\title{
Instability during bunch shortening of an electron-cooled beam
}

\author{
M. Takanaka \\ RIKEN (The Institute of Physical and Chemical Research), 2-1 Hirosawa, Wako-shi, 351-0198 Japan
}

(Received 1 November 2002; published 16 October 2003)

\begin{abstract}
Bunch shortening causes an electron-cooled beam to be space charge dominated at low energies. Instability during the bunch shortening has been studied using a particle-tracking program where the 3D space-charge field due to the beam is calculated with a simplifying model.
\end{abstract}

DOI: 10.1103/PhysRevSTAB.6.104201

PACS numbers: 29.27.Bd, 52.59.Sa

\section{INTRODUCTION}

The radioisotope (RI) beam factory [1] has a plan to have an electron-RI beam collider ( $e$-RI collider). Bunch shortening (BS) of an electron-cooled ion beam at low energies has been studied in order to satisfy ion-beam requirements at the collider, in performing particletracking simulations where the three-dimensional (3D) space-charge field is calculated $[2,3]$.

A longitudinal space-charge field in a beam in a perfectly conducting cylindrical chamber has been calculated by Nielsen et al. [4]. Longitudinal phenomena of bunched beams due to rf-wave-form distortion under the space charge and the reactive wall impedance have been described theoretically and experimentally by Hansen et al. [5]. The longitudinal phase-space envelope has been derived theoretically by Neuffer [6]. The longitudinal space-charge impedance has been found frequency dependent by Al-khateeb et al. [7]. In the following rings with electron-cooling systems installed, beams at low energies can be bunched to be space charge dominated. The longitudinal bunch profile has been observed to be parabolic in IUCF [8]. The longitudinal and the transverse characteristics of bunched beams have been studied in ESR [9]. Simulation codes [10] have been developed in order to study space-charge-dominated beams. The longitudinal field is calculated using $V_{\|}=-I Z_{\|}$in at least three of the codes as well as the early code [3], with $Z_{\|}$ being radially independent.

A novel simulation method where several sources of forces acting on a beam are taken into account is presented for the BS in Sec. II. Simulation results of a transverse instability during the BS are described and a cure for the instability is discussed before showing simulation results of the cure in Sec. III. Simulation results of a longitudinal instability during the BS are described and a cure for the instability is proposed in Sec. IV. The conclusions are summarized in Sec. V.

\section{SIMULATION METHOD}

In the simulation the following sources of forces acting on particles have been taken into account: (i) 3D spacecharge field induced by a beam itself; (ii) transverse dipole broadband impedance; (iii) electron cooling (EC) force [11]; (iv) solenoid field and toroid field of the EC section; (v) rf field for beam bunching; (vi) a uniform focusing section and a drift EC section to which the ring lattice is simplified. The ring parameters listed in Table I have been used as data input to the simulation.

The transverse charge distribution of a beam is assumed to be Gaussian with a standard deviation $\sigma$ which is much smaller than the radius of the vacuum chamber $b$. Then, the longitudinal monopole, dipole, and quadrupole space-charge impedances [12] are

$$
\begin{gathered}
{\left[\frac{Z_{\|}^{m}}{n}\right]_{s p}=i \frac{g^{m}(r)}{2 \beta \gamma^{2}} Z_{0},} \\
g^{0}(r) \approx-\int_{x=\frac{r^{2}}{2 \sigma^{2}}}^{\infty} \frac{e^{-x}}{x} d x+2 \log \left(\frac{b}{r}\right),
\end{gathered}
$$

TABLE I. Parameters of the ring, an initial coasting beam, and EC.

\begin{tabular}{lc}
\hline \hline \multicolumn{1}{c}{ Ring } & \\
\hline Circumference $2 \pi R$ & $180 \mathrm{~m}$ \\
Operation point $Q_{x} / Q_{y}$ & $4.425 / 3.725$ \\
Momentum compaction factor & $Q_{x}^{-2}$ \\
Chromaticities $\xi_{x} / \xi_{y}$ & $-25 /-30$ \\
Beta functions $\beta_{x} / \beta_{y}$ & $R / Q_{x} / R / Q_{y}$ \\
Dispersion $\eta_{x}$ & $R / Q_{x}^{2}$ \\
Twiss parameters at the EC section & \\
$\alpha_{x}^{e c}=\alpha_{y}^{e c}$ & 0 \\
$\beta_{x}^{e c}=\beta_{y}^{e c}$ & $7 \mathrm{~m}$ \\
$\eta^{e c}$ & $0 \mathrm{~m}$ \\
rf harmonics, or number of bunches $h$ & 60 \\
Inner radius of the vacuum chamber $b$ & $4 \mathrm{~cm}$ \\
\multicolumn{1}{c}{ Initial coasting beam } & \\
\hline Sixfold-rms momentum spread $6 \delta p / p$ & $10^{-3}$ \\
rms transverse emittances $\epsilon_{x}=\epsilon_{y}$ & $\pi \mathrm{m} \mathrm{rad}$ \\
& \\
\hline Electron-beam current & $4 \mathrm{~A}$ \\
Cathode temperature $k T_{c}$ & $0.1 \mathrm{eV}$ \\
Length of the EC section & $3 \mathrm{~m}$ \\
Electron-beam radius at the section & $0.1 \mathrm{Tm}$ \\
Solenoid field $B_{0}$ & \\
\hline \hline
\end{tabular}




$$
\begin{aligned}
g^{1}(r) \approx & \frac{2}{r} \operatorname{erf}\left(\frac{r}{\sqrt{2} \sigma}\right)-\frac{2}{\sigma} \sqrt{\frac{2}{\pi}} \exp \left(-\frac{r^{2}}{2 \sigma^{2}}\right) \\
& +\frac{2 r}{\sigma^{2}}\left[1-\operatorname{erf}\left(\frac{r}{\sqrt{2} \sigma}\right)\right]-\frac{2 r}{b^{2}} \\
g^{2}(r) \approx & -\left(\frac{1}{2 \sigma^{2}}+\frac{1}{r^{2}}\right) \exp \left(-\frac{r^{2}}{2 \sigma^{2}}\right)+\frac{1}{r^{2}} \\
& +\frac{r^{2}}{4 \sigma^{4}} \int_{x=\frac{r^{2}}{2 \sigma^{2}}}^{\infty} \frac{e^{-x}}{x} d x-\frac{r^{2}}{b^{4}}
\end{aligned}
$$

where $r$ is the radial position, $\beta$ and $\gamma$ the relativistic constants of the beam velocity $v, Z_{0}$ the impedance of the free space, $n=\left(\omega / \omega_{0}\right), \omega_{0}$ being the revolution frequency, $\sigma^{2}=\left(\sigma_{x}^{2}+\sigma_{y}^{2}\right) / 2$, and $\operatorname{erf}(x)$ the error function.

The longitudinal field is described approximately as

$$
\frac{i}{2 \pi} \sum_{m=0}^{2}\left[\frac{Z_{\|}^{m}}{n}\right]_{s p} \frac{d I^{m}}{d s},
$$

where $s$ is the longitudinal coordinate, $I^{0}$ the instantaneous current or $v \lambda(s), I^{1} v \lambda(s)(\bar{x} x+\bar{y} y) / r$, and $I^{2}$ $v \lambda(s)\left(2 x^{2} / r^{2}-1\right)\left(\sigma_{x}^{2}-\sigma_{y}^{2}\right) / 2$, with $\lambda$ being the charge line density.

It is said [7] that the space-charge impedance attenuates to become half the value at $0 \mathrm{~Hz}$ around $14-19 \mathrm{GHz}$ for the beam size $3-4 \mathrm{~mm}$ in the current simulation condition. Thus, the monopole, dipole, and quadrupole impedances are assumed to attenuate exponentially beyond $10 \omega_{c} \simeq 12 \mathrm{GHz}$ :

$$
\exp \left(1-\frac{\Omega}{10 \omega_{c}}\right)
$$

where $\omega_{c}=c / b$ is the characteristic resonance frequency for the broadband impedance model (or about half the cutoff frequency for the $H_{11}$ mode in the cylindrical vacuum chamber), with $c$ being the light velocity.

The transverse field is calculated using the charge distribution on transverse $101 \times 101$ cells across the cross section of $(80 \mathrm{~mm})^{2}$ of which only the central cell consists of other $13 \times 13$ cells in itself. Since the simulation has shown that the cooled beam is extremely centralized transversely even when it becomes space charge dominated, such a twofold cell structure has been used eventually. Seven field maps are made for seven divisions along the bunch, the structure being formed at each division. The field at any particle is estimated making the field interpolation between two neighboring maps and taking account of the charge line density $\lambda(s)$. The field calculation contains contributions from image charges reflected from the perfectly conducting smooth wall of the vacuum chamber [13]. The magnetic-field contribution is taken into account with a factor $1 / \gamma^{2}$.

The transverse field due to the broadband impedance model is calculated using the following wake function $W_{1}(z)$ [12] on the assumption of the longitudinal mono- pole broadband impedance $\left|Z_{\|}^{B B} / n\right|$ of $50 \Omega$ :

$$
\begin{gathered}
W_{1}(z)=\frac{v R_{s \perp} \omega_{c}}{Q \bar{\omega}} e^{\alpha z / v} \sin \frac{\bar{\omega} z}{v}, \\
R_{s \perp}=\frac{2 Q}{b^{2}} \frac{\omega_{c}}{\omega_{0}}\left|\frac{Z_{\|}^{B B}}{n}\right|,
\end{gathered}
$$

where the relation between the transverse dipole broadband impedance and the longitudinal monopole broadband impedance [14] has been used, $\alpha=$ $\left(\omega_{c} / 2 Q\right), \bar{\omega}=\sqrt{\omega_{c}^{2}-\alpha^{2}}, z(<0)$ is the longitudinal separation from a wake-source point to the wake-field point, and $Q$ is assumed to be 1 . The wake field is neglected when $z$ is behind two bunch spacings.

A parabolic-bunch length of a space-charge-dominated beam $l$ depends on rf voltage $V_{\mathrm{rf}}$ and the harmonics $h$ when the energy gain from the rf field is compensated by that from the longitudinal space-charge field:

$$
l^{3} \propto \frac{1}{h V_{\mathrm{rf}}} .
$$

Fundamental $\mathrm{rf}$ voltage is applied in order to bunch a coasting cooled beam. A 3rd-harmonic one is applied additionally when the beam bunched shorter than onethird the bunch spacing is shortened further. The two rf cavities installed at an azimuth are located at the azimuth $\pi$ from the EC section.

Since a single bunch phenomenon during the BS is an exclusive interest, every bunch is assumed to behave the same. The time step of the particle tracking is $1 / 160$ of the revolution time. Using 40000 macroparticles, the BS process has been simulated for a ${ }^{238} \mathrm{U}^{92+}$ beam of $4 \mathrm{~mA}$, or $5.4 \times 10^{6}$ ions per bunch at $150 \mathrm{MeV} / \mathrm{u}$.

Under the parameters of the EC system listed in Table I the cooling time for a coasting ${ }^{238} \mathrm{U}^{92+}$ beam of $0 \mathrm{~A}$ has been estimated at $5 \mathrm{~ms}$ for the longitudinal direction and $17 \mathrm{~ms}$ for the transverse direction by performing the simulation.

\section{TRANSVERSE INSTABILITY}

A coasting beam precooled in an accumulator cooler ring is injected into the $e$-RI collider and is cooled and bunched using the EC and the rf system as follows. First, the coasting beam is cooled to the sixfold-rms momentum spread of $5 \times 10^{-4}$. Next, the fundamental rf voltage is increased from $0 \mathrm{~V}$ in such a way that the momentum spread measured at the EC section is maintained at $5 \times$ $10^{-4}$. When the sixfold-rms bunch length reaches onethird the bunch spacing $(=1 \mathrm{~m})$, the increase stops. Then, after the momentum spread is cooled to $1.9 \times$ $10^{-4}$ or most of the bunched beam gathers within $1 \mathrm{~m}$, the 3rd-harmonic rf voltage is increased from $0 \mathrm{~V}$ keeping the momentum spread. Simulation results of the process in Fig. 1 show that a transverse coherent oscillation grows still after fixing the rf voltages and leads the beam 

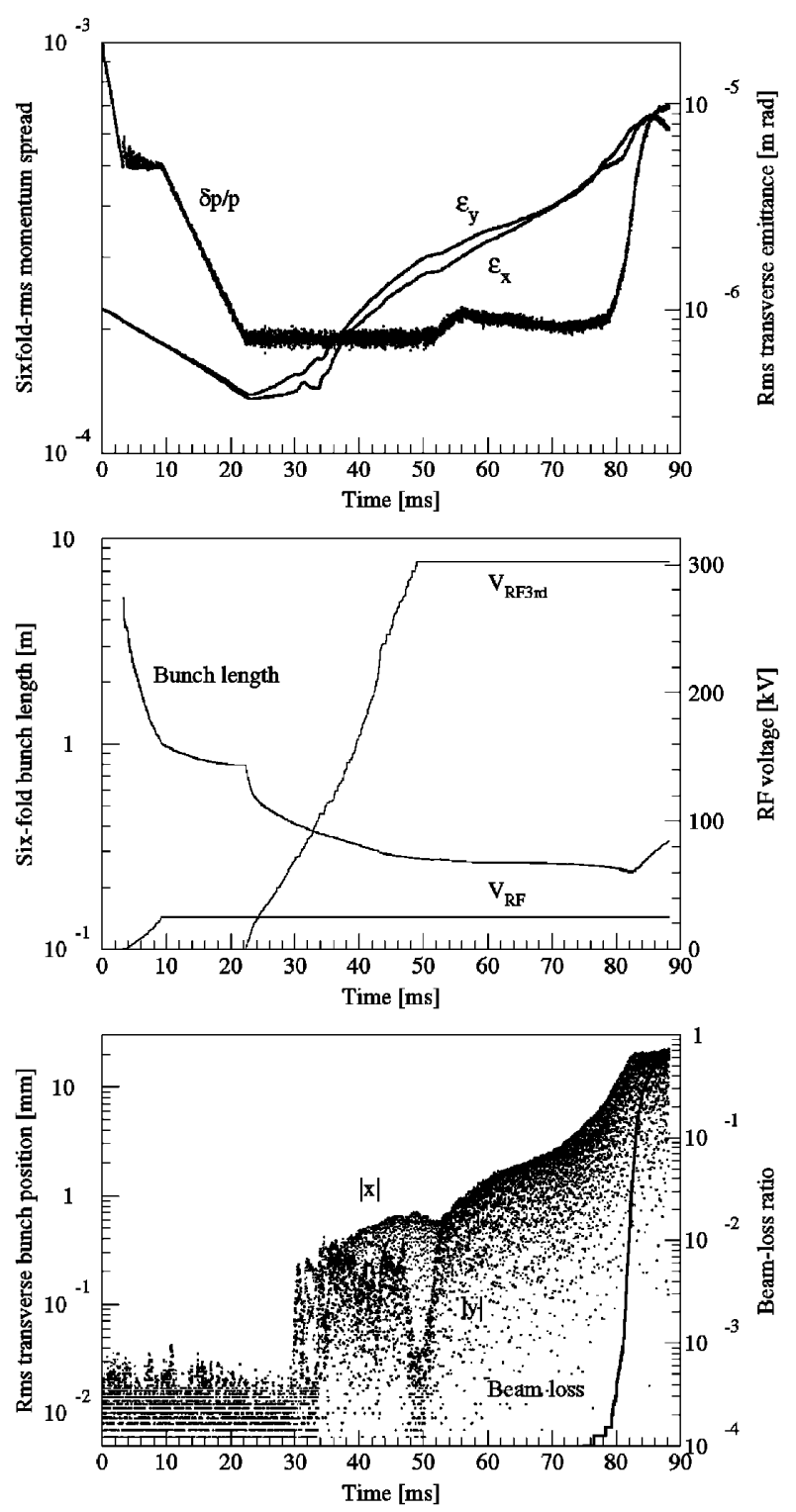

FIG. 1. Time evolution of the ${ }^{238} \mathrm{U}^{92+}$ beam of $4 \mathrm{~mA}$ during the BS.

to a fatal beam loss through hitting the vacuum chamber. Figure 2 shows that the transverse dipole-mode oscillation starts at the bunch center.

\section{A. Source of the transverse instability}

Passing through the EC section the ions receive not only the EC force, the cyclotron-motion force due to the solenoid field $B_{0}$, and the focusing force due to the electron beam, but also the force due to the toroid field at both ends of the section. Since a simulation where the effect of the toroid field is neglected has shown that the transverse instability never occurs during the BS, the field can be regarded as a source of the instability.
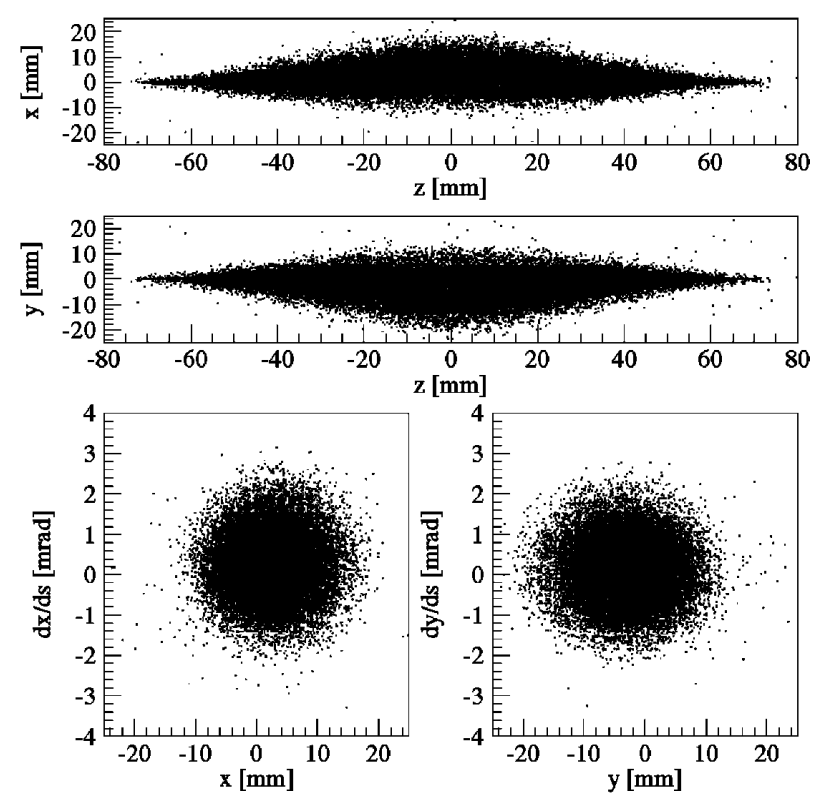

FIG. 2. Charge distribution of the unstable beam snapshot at the EC section at $69 \mathrm{~ms}$.

In order to estimate the toroid field, the following structure of the toroid of the horizontally aligned EC device, as shown in Fig. 3, is assumed. The transverse cross section of the toroid is equal to that of the solenoid, the radial center of the toroid is at $x_{t}$ from the $s$ axis, and the radius of the curvature of the outside wall is $r_{t}$. There is the field transition around the joint between the solenoid and the toroid. Then, the field in the toroid is $B_{0} x_{t} / \sqrt{s^{2}+\left(x_{t}-x\right)^{2}}$. When ions pass in parallel with

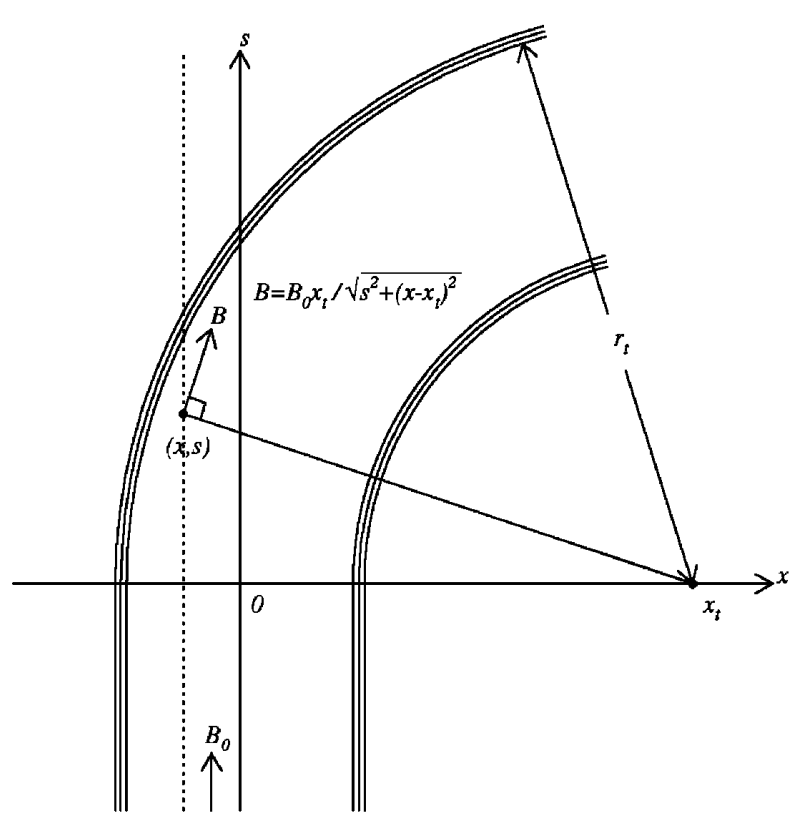

FIG. 3. Structure of the toroid idealized for estimation of the toroid field. 
the $s$ axis through the toroid, the integration of the horizontal component of the field over the path is approximately

$$
B_{0} x_{t} \log \frac{r_{t}}{x_{t}}+B_{0} x
$$

The first term expresses an effect of bending beams vertically and the second one an effect of twisting the beams. After ideal compensation of the bending effect using steering magnets, passing through the toroid the ions receive the following vertical kick:

$$
\Delta\left(\frac{d y}{d s}\right)=-\frac{q B_{0} x}{p},
$$

where $q$ is the charge of the ions and $p$ the momentum. The kicks are opposite at both the ends. It is seen below that $Q_{x} \pm Q_{y}=n(n=$ integer $)$ resonances are induced by the kicks.

\section{B. Control of the transverse-instability source}

Resonances due to the twisting effect and the resonance control by using skew quadrupole magnets (SQM's) are described. The amplitude and the phase equation for a particle are given as follows when toroids and SQM's are treated as thin lenses:

$$
\begin{aligned}
& \frac{d \epsilon_{x, y}}{d \theta}=-2 Q_{\underline{x, y}} \epsilon_{x, y}^{1 / 2} \sum_{i} \beta_{\underline{x, y}}^{3 / 2} g_{\underline{x, y}}^{i} \sin \Phi_{\underline{x, y},} \\
& \frac{d \Phi_{x, y}}{d \theta}=Q_{\underline{x, y}}-Q_{\underline{x, y}} \epsilon_{\underline{x, y}}^{-1 / 2} \sum_{i} \beta_{\underline{x, y}}^{3 / 2} g_{\underline{x, y}}^{i} \cos \Phi_{\underline{x, y}}, \\
& g_{\underline{x, y}}^{i}=\frac{K_{\underline{y, x}}^{i} l^{i}}{Q_{x, y} \beta_{x, y}^{i}} \delta\left(\theta-\theta_{\underline{x, y}}^{i}\right) \underline{y, x}
\end{aligned}
$$

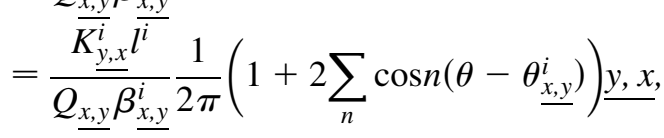

$$
\begin{aligned}
& \underline{x, y}=\sqrt{\underline{\epsilon_{x, y} \beta_{x, y}}} \cos \Phi_{\underline{x, y}} \\
& K_{x}^{i} l^{i}= \pm \frac{q B_{0}}{p}, \quad K_{y}^{i} l^{i}=0 \quad \text { for toroids, } \\
& K_{\underline{y, x}}^{i} l^{i}=\frac{q \frac{\partial B_{y}^{i}}{\partial x} l^{i}}{p} \quad \text { for SQM's, }
\end{aligned}
$$

where the description is based on the transverse normalized phase spaces [15], the suffix $i$ is for toroids and SQM's, $\phi_{x, y}$ being an offset, $\Phi_{x, y}=Q_{x, y} \theta+\phi_{x, y}$ is the phase on the phase space, and $x, y$ or $y, x$ means that it has to be $x$ or $y$ on the same side with respect to the commas in an equation.

When only main contributions to slowly varying terms $\left(Q_{x}+Q_{y}=n\right.$ resonance terms) are taken into account, the above differential equations can be rewritten as

$$
\begin{aligned}
& \frac{d \epsilon_{x, y}}{d \theta} \approx-\frac{1}{2 \pi} \sqrt{\epsilon_{x} \epsilon_{y}} A_{\underline{x, y}} \sin \left(\Phi+n \theta_{\underline{x, y}}^{\text {eff }}\right),
\end{aligned}
$$

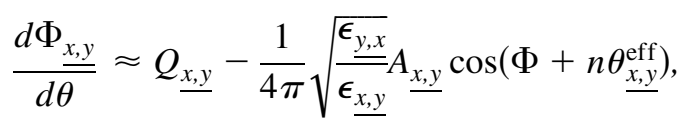

$$
\begin{aligned}
& A_{\underline{x, y}}^{2} \equiv\left(\sum_{i} \sqrt{\beta_{x}^{i} \beta_{y}^{i}} K_{\underline{y, x}}^{i} l^{i} \cos n \theta_{\underline{x, y}}^{i}\right)^{2} \\
& +\left(\sum_{i} \sqrt{\beta_{x}^{i} \beta_{y}^{i}} K_{\underline{y, x}}^{i} l^{i} \sin n \theta_{\underline{x, y}}^{i}\right)^{2}, \\
& \sin n \theta_{\underline{x, y}}^{\mathrm{eff}} \equiv \frac{\sum_{i} \sqrt{\beta_{x}^{i} \beta_{y}^{i}} K_{\underline{y, x}}^{i} l^{i} \sin n \theta_{\underline{x, y}}^{i}}{A_{\underline{x, y}}}, \\
& \Phi=\Phi_{x}+\Phi_{y}-n \theta .
\end{aligned}
$$

\section{Resonance due to the toroids}

In the case where only the toroids are on, the above equations are

$$
\begin{gathered}
\frac{d \epsilon_{x}}{d \theta}=0, \quad \frac{d \Phi_{x}}{d \theta}=Q_{x}, \\
\frac{d \epsilon_{y}}{d \theta}=-\frac{1}{2 \pi} \sqrt{\epsilon_{x} \epsilon_{y}} A_{y \text { tor }}^{\text {eff }} \sin \left(\Phi+n \theta_{y \text { tor }}^{\text {eff }}\right) \\
\frac{d \Phi_{y}}{d \theta}=Q_{y}-\frac{1}{4 \pi} \sqrt{\frac{\epsilon_{x}}{\epsilon_{y}}} A_{y \text { tor }}^{\text {eff }} \cos \left(\Phi+n \theta_{y \text { tor }}^{\text {eff }}\right),
\end{gathered}
$$

where $A_{y \text { tor }}^{\text {eff }}$ is $A_{y}$ for the case where $\left(\partial B_{y}^{i} / \partial x\right)=0$.

The following resonant invariants are derived from the above equations:

$$
\begin{gathered}
\epsilon_{x}=C, \\
\left(Q_{x}+Q_{y}-n\right) \epsilon_{y}-\frac{1}{\pi} \sqrt{\epsilon_{x} \epsilon_{y}} A_{y \text { tor }}^{\mathrm{eff}} \cos \left(\Phi+n \theta_{y \text { tor }}^{\mathrm{eff}}\right)=C .
\end{gathered}
$$

The above equations show that the beam can be trapped into an emittance-increasing $Q_{x}+Q_{y}=n$ resonance while the horizontal emittance is fixed, as shown in Fig. 4. The half stop band width is defined as $\sqrt{\epsilon_{x} / \epsilon_{y}} A_{y \text { tor }}^{\text {eff }} / 4 \pi$.

The type of the $Q_{x}-Q_{y}=n$ resonance due to the toroid is an emittance-increasing one, too.

\section{Resonance control}

$\theta_{x}^{i}$ is nearly equal to $\theta_{y}^{i}$ especially in the current simulation where beta functions are constant along the uniform focusing section. $A_{y}$ includes terms of toroids and 


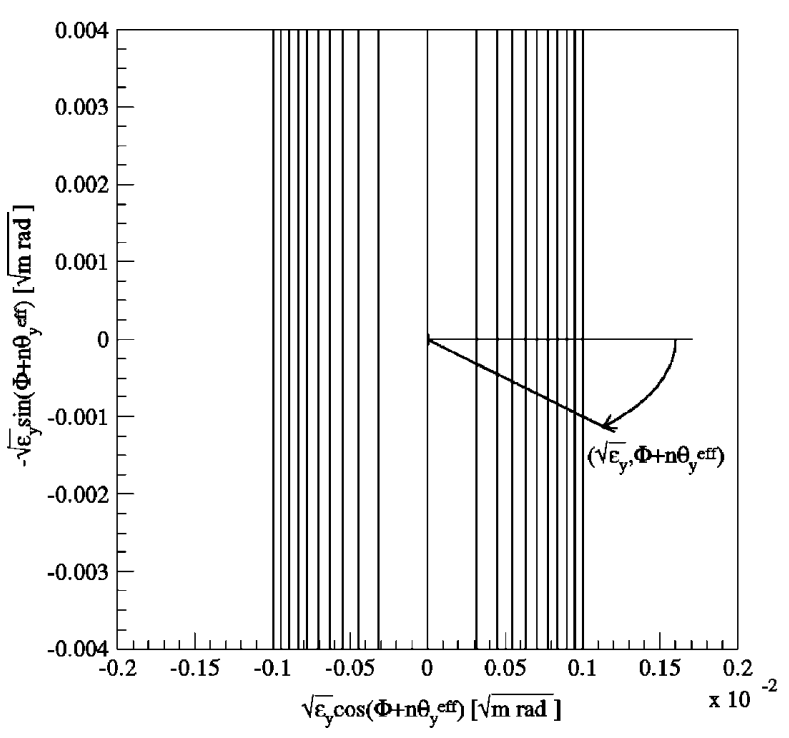

FIG. 4. Resonant invariant for the emittance-increasing resonance due to the toroid in the initial case where $\epsilon_{y} \leq 1 \times$ $10^{-6} \mathrm{~m} \mathrm{rad}$ and $Q_{x}+Q_{y}=n$.

SQM's, while $A_{x}$ includes only terms of SQM's. It is difficult to make $A_{y}$ and $A_{x}$ zero simultaneously using some SQM's, or to suppress the resonance. It is easy, however, to make $A_{x}=A_{y} \approx A_{y \text { tor }}^{\text {eff }} / 2$ and $n \theta_{x}^{\text {eff }}-n \theta_{y}^{\text {eff }}=$ $m \pi$ ( $m=$ odd integer). In the case, the amplitude and the phase equation are

$$
\begin{gathered}
\epsilon_{x}+\epsilon_{y}=\epsilon=C \\
\frac{d \epsilon_{y}}{d \theta}=-\frac{1}{2 \pi} \sqrt{\left(\epsilon-\epsilon_{y}\right) \epsilon_{y}} A_{y} \sin \left(\Phi+n \theta_{y}^{\mathrm{eff}}\right), \\
\frac{d \Phi}{d \theta}=Q_{x}+Q_{y}-n \\
-\frac{1}{4 \pi} \frac{\epsilon-2 \epsilon_{y}}{\sqrt{\left(\epsilon-\epsilon_{y}\right) \epsilon_{y}}} A_{y} \cos \left(\Phi+n \theta_{y}^{\mathrm{eff}}\right) .
\end{gathered}
$$

The following resonant invariant is derived from the above equations:

$$
\left(Q_{x}+Q_{y}-n\right) \epsilon_{y}-\frac{1}{\pi} \sqrt{\left(\epsilon-\epsilon_{y}\right) \epsilon_{y}} A_{y} \cos \left(\Phi+n \theta_{y}^{\text {eff }}\right)=C .
$$

The above equation shows that the beam can be trapped in an emittance-beating $Q_{x}+Q_{y}=n$ resonance, as shown in Fig. 5.

It can be expected that the transverse instability can be cured by changing the $Q_{x}+Q_{y}=n$ resonance from the emittance-increasing type to an emittance-beating one using SQM's.

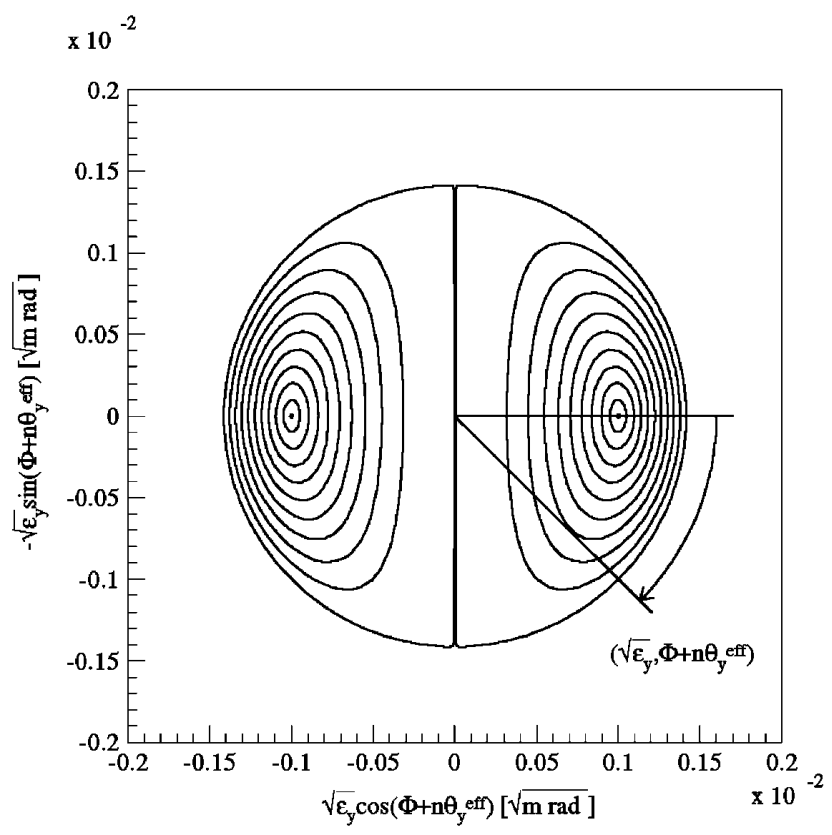

FIG. 5. Resonant invariant for the emittance-beating resonance controlled using SQM's in the initial case where $\epsilon_{x}+$ $\epsilon_{y}=2 \times 10^{-6} \mathrm{~m}$ rad and $Q_{x}+Q_{y}=n$.

\section{Cure for the transverse instability}

Figure 6 shows that just before the transverse instability occurs the beam is across the $Q_{x} \pm Q_{y}=n$ resonance lines, $70 \%$ of the beam being below the major $Q_{x}+Q_{y}=$ 8 resonance line (the nearest to and below the operation point). As the simplest cure, however, two SQM's are used in order to realize the emittance-beating type of the major resonance and the zero stop band width for the $Q_{x}=Q_{y}$ resonance. $\theta_{x}^{i}$ and $\theta_{y}^{i}$ at $Q_{x}+Q_{y}=n$ are

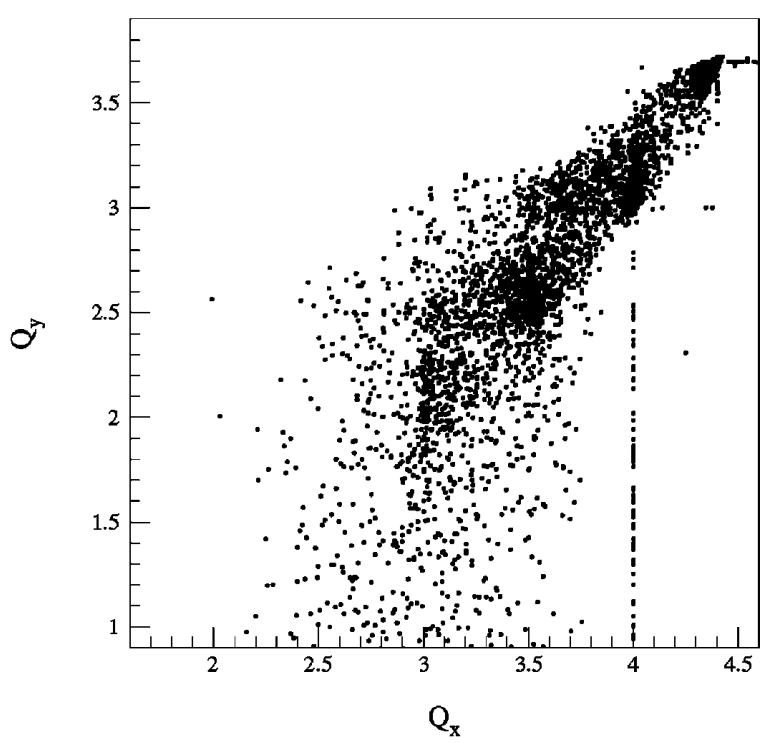

FIG. 6. Incoherent betatron-tune distribution just before the transverse instability (at $28 \mathrm{~ms}$ in Fig. 1). 
estimated on the assumption that the phase advance $\mu_{0}$ and the beta function $\beta_{0}$ at the operation point $\left(Q_{x 0}, Q_{y 0}\right)$ are changed under the space-charge effects as follows:

$$
\begin{aligned}
& \mu=\frac{n}{Q_{x 0}+Q_{y 0}} \mu_{0}, \\
& \beta=\frac{Q_{x 0}+Q_{y 0}}{n} \beta_{0} .
\end{aligned}
$$

The SQM's with $\left(\partial B_{y} / \partial x\right) l= \pm 0.084 \mathrm{~T}$ are installed at the dispersion-free points (the azimuths $\pm 0.86 \pi$ from the EC section, respectively) in the simulation. No care of the stop band widths of the other resonances is taken: after (or before) the resonance control the widths of the $Q_{x}+$ $Q_{y}=7,6$, and $Q_{x}-Q_{y}=1$ resonance are $0.002,0.015$,
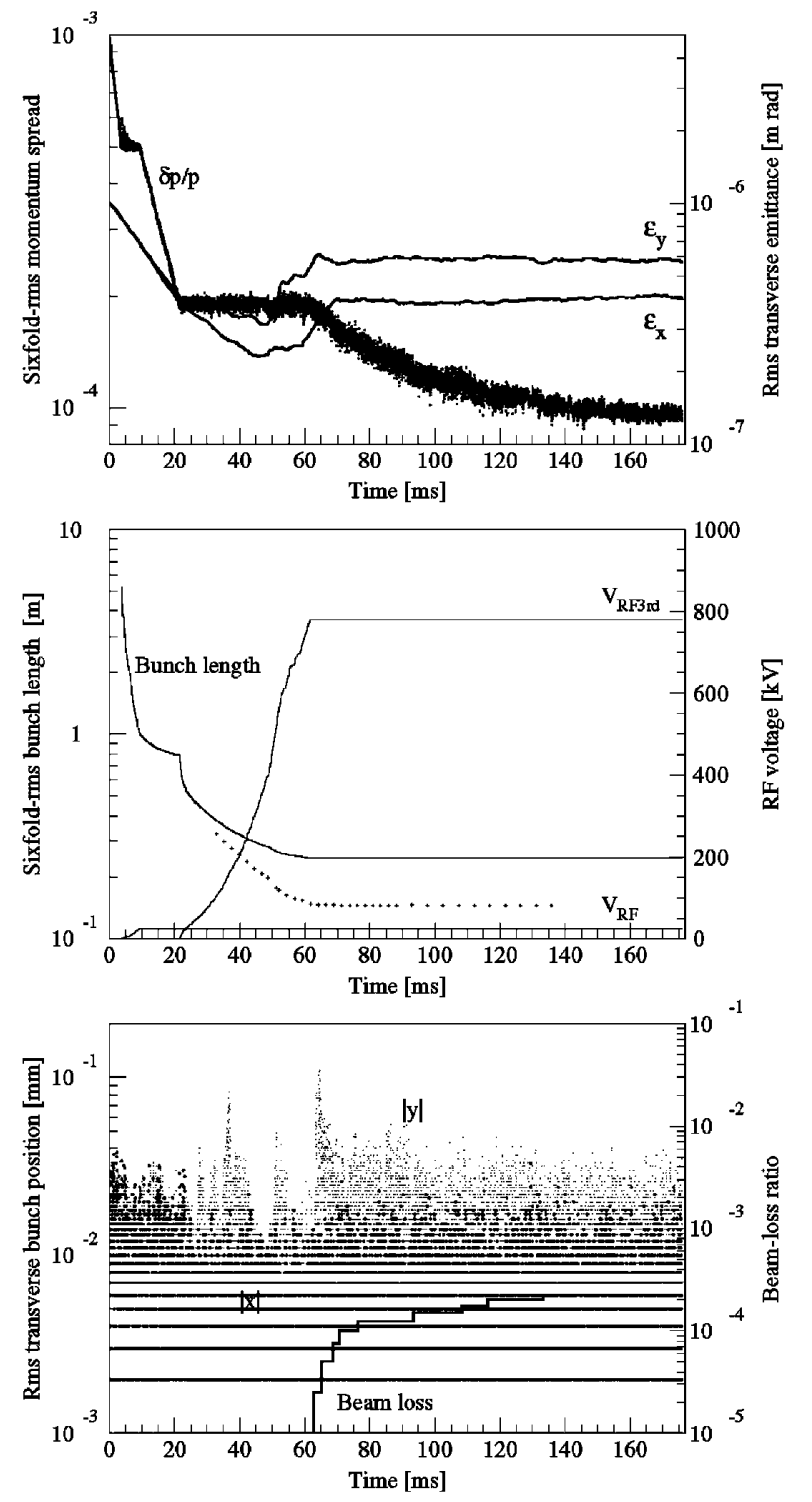

FIG. 7. Time evolution of the beam under the resonance control. In the middle panel the dotted curve indicates the sixfold-rms bunch length without counting ions at $z= \pm 1 \mathrm{~m}$. and 0.004 (or $0.005,0.005$, and 0.0005), respectively. Simulation results of the resonance control in Fig. 7 show that the resonance control is effective for the transverse-instability cure. After $160 \mathrm{~ms}$ the beam reaches the equilibrium among EC, the space-charge effects, the rffield effect, and the resonance effect due to the imperfection of the resonance control.

\section{LONGITUDINAL INSTABILITY}

When the bunch is further shortened by applying a higher 3rd-harmonic rf voltage, a longitudinal instability starts with a sudden increase in the momentum spread and continues in oscillation in the momentum spread, as shown in Fig. 8. During the instability the bunch length
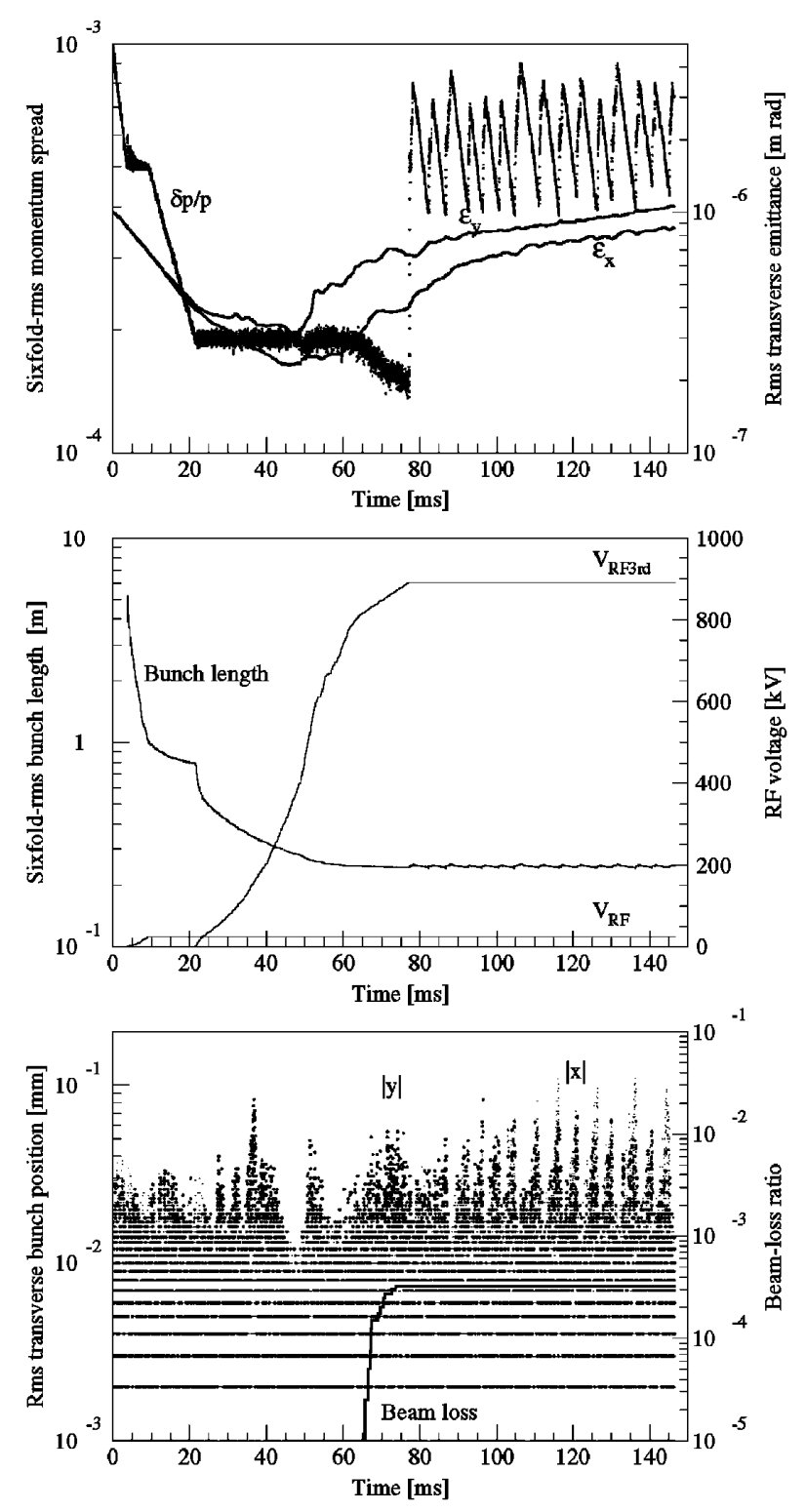

FIG. 8. Time evolution of the beam under the resonance control with a higher 3rd-harmonic rf voltage applied. 

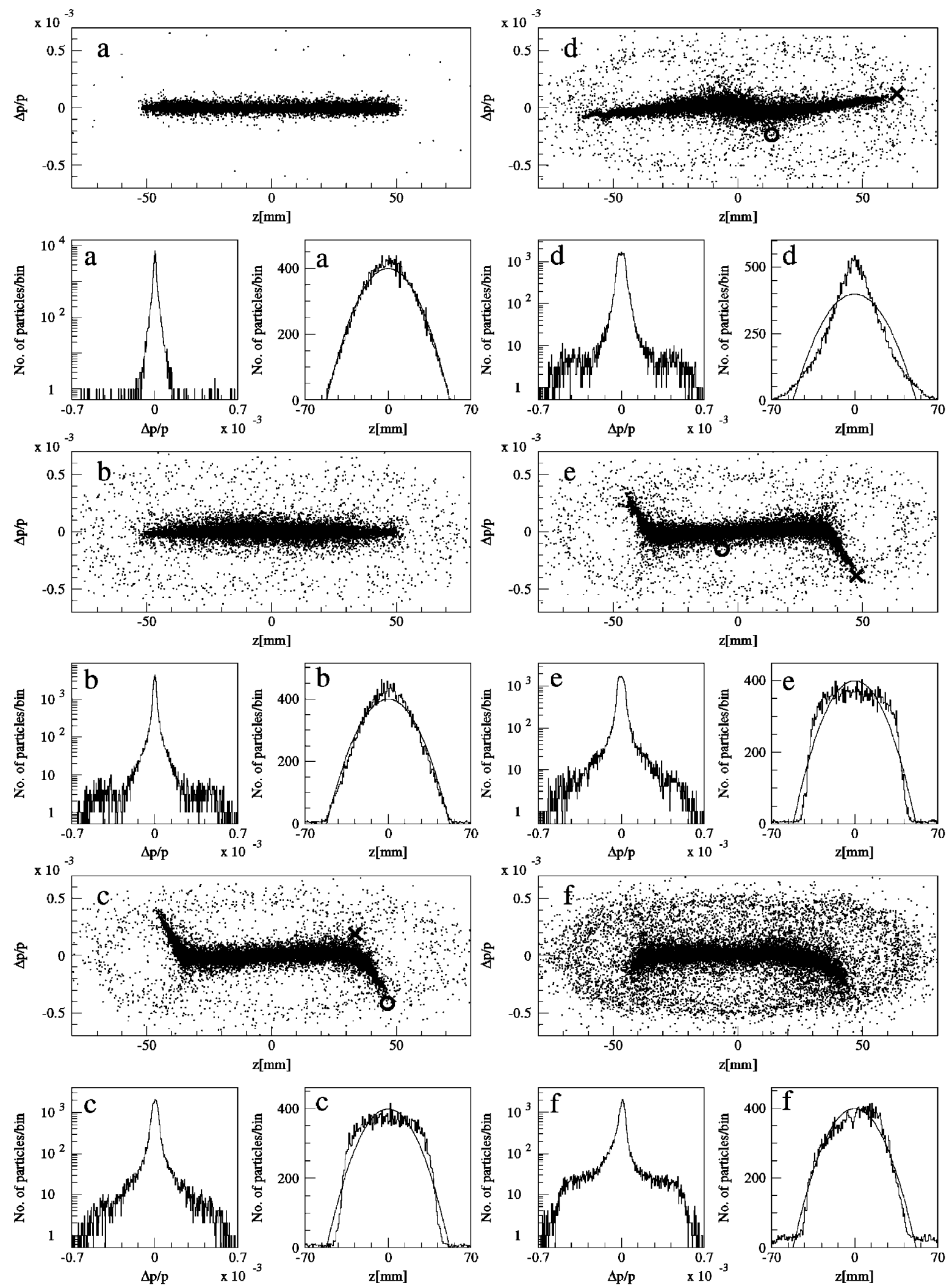

FIG. 9. Longitudinal phase-space distributions during the longitudinal instability. The figures (a) are ones just before the longitudinal instability or at $76.1 \mathrm{~ms}$ when the momentum spread $(6 \delta p / p)$ is $1.5 \times 10^{-4}$. The figures (b) are ones at $116.2 \mathrm{~ms}$, the figures (c)-(e) snapshots taken at each revolution interval at $116.4 \mathrm{~ms}$, and the figures (f) ones at $117.3 \mathrm{~ms}$, when $6 \delta p / p$ is $3.9 \times 10^{-4}$ at the minimum, $5.0 \times 10^{-4}$, and $7.8 \times 10^{-4}$ at the maximum, respectively. The parabolic curve fitted to the bunch profile (a) is drawn in (a)-(f). Two particles are marked with $\bigcirc$ and $\times$, respectively, in (c) - (e). 
does not increase while the transverse emittances increase slowly.

\section{A. Characteristics}

Just before the longitudinal instability happens, the momentum spread is $1.5 \times 10^{-4}$. After the sudden increase in the momentum spread at $77.3 \mathrm{~ms}$, the momentum spread oscillates between $4 \times 10^{-4}$ and $9 \times 10^{-4}$ at the fast speed for the increase or at the slow speed for the decrease.

The longitudinal phase-space distributions shown in Fig. 9 are ones that are snap shot at the EC section. There is little halo around the phase-space distribution just before the longitudinal instability, as shown in Fig. 9(a). The parabolic curve fitted to the bunch profile in Fig. 9(a) is drawn in Figs. 9(a)-9(f) as a marker of the equilibrium between the longitudinal space-charge effect and the rffield effect.

At the times of the minimum momentum spread during the instability, the phase-space distribution consists of a core and a halo in symmetry, as shown in Fig. 9(b). With the synchrotron oscillation being clockwise on the phase space, the synchrotron tune along the outer edge of the halo is 0.15 that is close to the bare tune of 0.16 . The nearer to the core, the closer to zero the tune is because of the rf-wave-form distortion due to the longitudinal spacecharge effect.

At the times of the momentum spread of $0.5 \times 10^{-3}$ on the way to the maximum momentum spread, the phasespace distribution looks like ones shown in Figs. 9(c)9(e) that are a series of snapshots taken at each revolution interval. The core is seen to lengthen and shorten once for two revolutions. For the shortened (or lengthened) core the line density at the center, the head, and the tail of the core is lower (or higher) than before the instability. Therefore, when the phase-space distribution shown in Fig. 9(b) is considered to be a monopole mode, the density oscillation can be explained as the rotation of an octupole-mode distribution around the monopole mode. Figure 10 shows that the frequency spectrum of the monopole moment along the bunch is characterized by the following signals: (i) along $\Omega / \omega_{0}=k h$ signals due to the monopole; (ii) along $\Omega / \omega_{0}=k h+(i / 2) \quad(i=$ integer) signals due to the octupole; (iii) along $\Omega / \omega_{0}=$ $k h+(i / 4)$ signals due to the quadrupole; (iv) along $\Omega / \omega_{0}=k h+i(i=$ nonzero integer $)$ signals due to a balance between the momentary energy gain from the rf field and the durable energy gain from the longitudinal space-charge field during a revolution; (v) reflection signals from the longitudinal space-charge impedance. The spectrum shows that the oscillation mode of the phasespace distribution is mainly quadrupole and octupole at the tune of 0.125 , which is seen from Figs. 9(c) $-9(\mathrm{e})$, too.

When the absolute value of the gradient of the beam line density is larger (or smaller) than that of the parabolic curve, the space-charge effect of counterclockwise

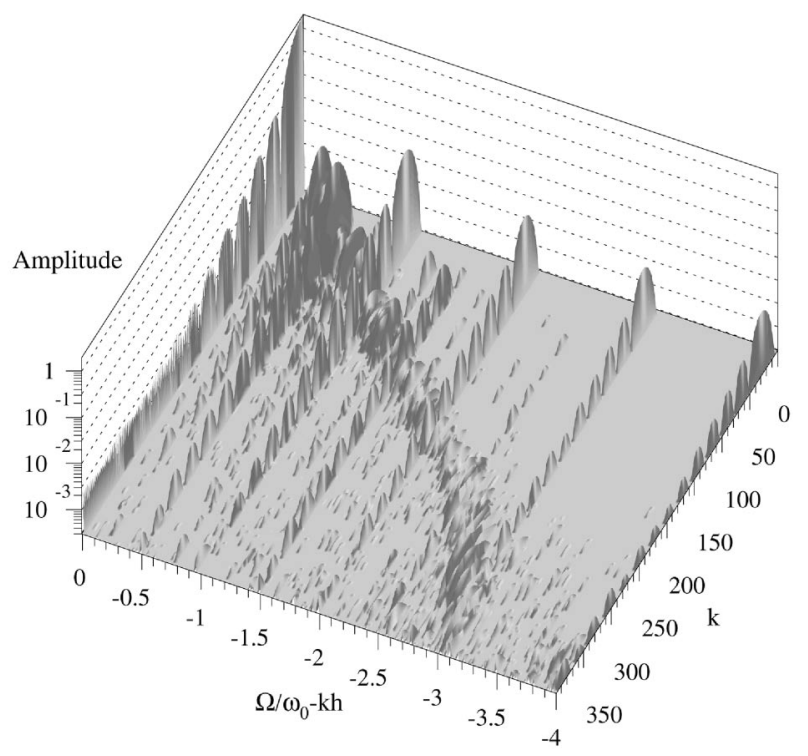

FIG. 10. Frequency spectrum of the monopole moment on the way to the maximum momentum spread at $116.4 \mathrm{~ms}$ during the longitudinal instability. For the sake of the clear sight of the main signals, the spectrum normalized by the dc component is cut away below $3 \times 10^{-4}$.

bunch rotating (or the rf-field effect of clockwise bunch rotating) appears locally on the phase-space distribution after a revolution, as shown in Figs. 9(c)-9(e).

At the times of the maximum momentum spread the phase-space distribution is one with the dense halo, as shown in Fig. 9(f). Although the distribution is not always asymmetrical, the frequency spectrum of the monopole moment along the bunch reveals that the oscillation modes of the distribution are dipole to decapole at the tune of 0.1 . The bunch length becomes $16 \mathrm{~cm}$ because of the growth of the halo, while the bunch length is $10.2 \mathrm{~cm}$ just before the instability.

\section{B. Cure for the longitudinal instability}

After the 3rd-harmonic rf voltage reaches $780 \mathrm{kV}$, the 9th-harmonic rf voltage is applied at the same azimuth in the ring as the 3rd-harmonic one. The simulation results of the application in Fig. 11 show that the beam is stable longitudinally under the 9th-harmonic rf voltage of $160 \mathrm{kV}$. The $160 \mathrm{kV}$ corresponds to an addition of 480 to $780 \mathrm{kV}$ of the 3rd-harmonic rf voltage. The sum of these two voltages is beyond the longitudinal-instability threshold of $0.9 \mathrm{MV}$. The bunch length is shortened $9.0 \mathrm{~cm}$.

A line density of a stable space-charge-dominated bunched beam has the following approximate relation with the $m$ th-harmonic rf voltage when no other rf voltage is applied:

$$
\frac{d \lambda}{d z} \propto-\sin \frac{h m z}{R},
$$



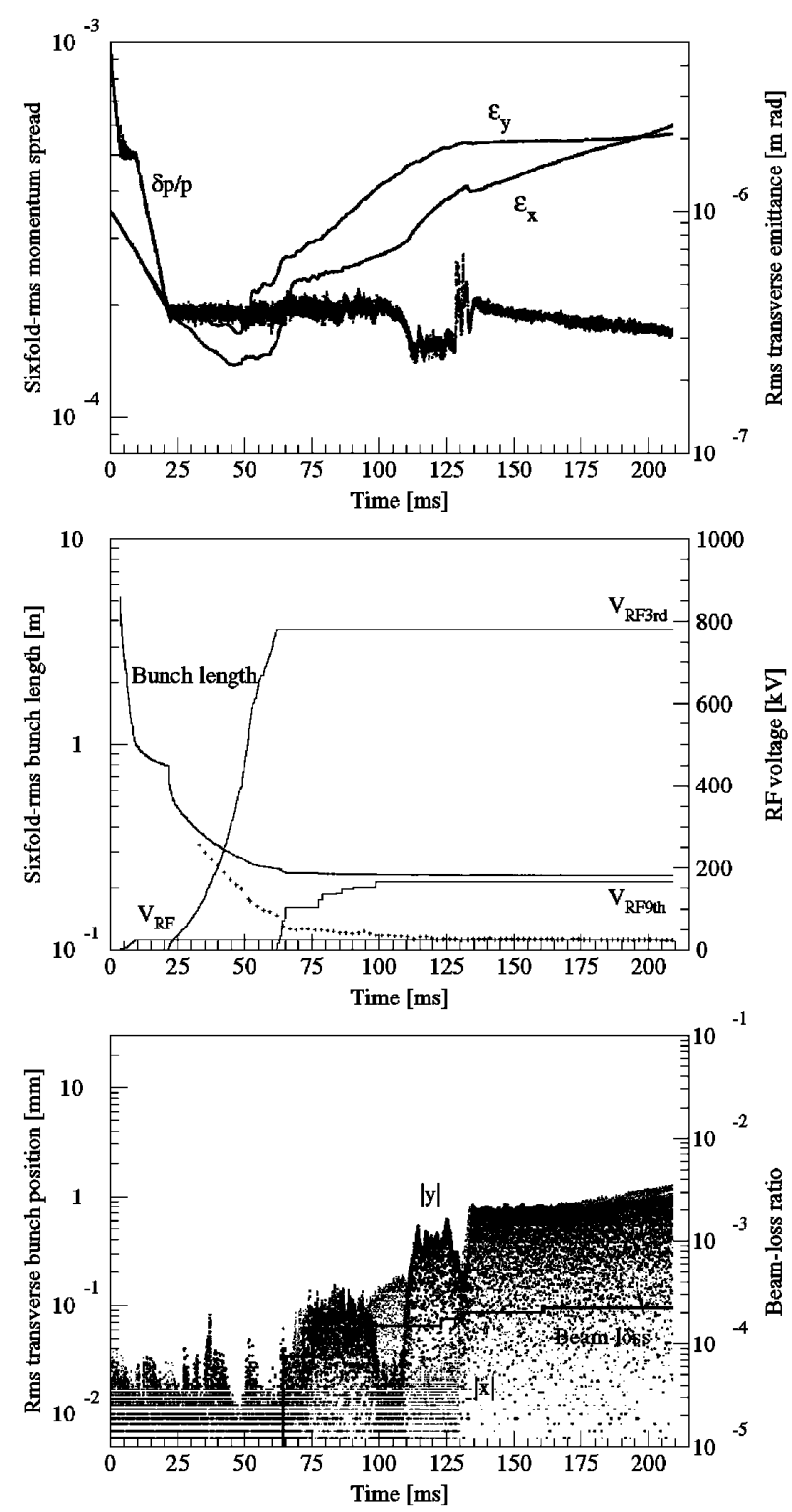

FIG. 11. Time evolution of the beam under the resonance control with the 9th-harmonic rf voltage applied additionally. In the middle panel the dotted curve indicates the sixfold-rms bunch length without counting the ions at $z= \pm 1 \mathrm{~m}$.

$$
\lambda \propto 1-\frac{4 z^{2}}{l_{0}^{2}}+\frac{h^{2} m^{2} z^{4}}{3 R^{2} l_{0}^{2}},
$$

where $l_{0}$ is the parabolic-bunch length when the nonlinearity of the voltage around the zero cross is negligibly small. The nonlinearity has an effect that makes the bunch confinement loose on the head and the tail side. The effect due to the 9th-harmonic rf voltage is 9 times stronger at the bunch ends than that due to the 3rdharmonic one when the 9th-harmonic one is one-third of the 3rd-harmonic one, or when the $l_{0}$ is equal under both [see Eq. (8)]. As the simulation result, the nonline- arity of the 9th-harmonic rf voltage is seen to play a role in cure for the longitudinal instability.

The beam, however, is exposed to another transverse instability whose growth time is much longer than that of the previous one shown in Fig. 1. The instability has been checked to occur without the transverse broadband impedance or without consideration of image charges reflected from the chamber in the simulation. It is guessed that the weak instability is due to the imperfection of the resonance control.

\section{SUMMARY}

In order to study fundamental characteristics of an electron-cooled beam during BS, particle-tracking simulations have been performed where the 3D space-charge field due to the beam is calculated with a simplifying mode and a ring lattice is simplified. The simulation results show the following three points:

The cooled beam encounters a transverse and a longitudinal instability during BS.

The transverse instability which leads to a fatal beam loss can be cured by controlling the $Q_{x} \pm Q_{y}=n$ resonances due to a gradient component of the toroid field at the EC section using skew quadrupole magnets.

The longitudinal instability which slowly increases the transverse emittances can be cured by additionally applying higher-order-harmonic rf voltage.

\section{ACKNOWLEDGMENTS}

The author would like to thank Professor T. Katayama for giving him a motive for the current study. The author thanks Dr. A. Goto for purchasing the fastest Digital Alpha computers in the early years of the study just for the simulation calculation.

[1] Y. Yano et al., in Proceedings of the 2001 PAC, Chicago, IL (IEEE, Piscataway, NJ, 2001), p. 575.

[2] M. Takanaka, in Proceedings of the 2001 PAC (Ref. [1]), p. 3897; RIKEN Report No. RIKEN-AF-AC-29, 2001; in Proceedings of the 13th Symposium on Accelerator Science and Technology, Osaka, 2001 (RCNP, Osaka, 2001), p. 89; in Proceedings of the EPAC2002, Paris (EPS-IGA, Geneva, 2002), p. 1410.

[3] M. Takanaka and T. Katayama, in Proceedings of the 1997 PAC, Vancouver, B.C. (IEEE, Piscataway, NJ, 1998), p. 1807.

[4] C. E. Nielsen, A. M. Sessler, and K. R. Symon, in Proceedings of the International Conference on High Energy Accelerators and Instrumentation, CERN (CERN, Geneva, 1959), p. 239.

[5] S. Hansen et al., IEEE Trans. Nucl. Sci. 23, No. 3, 1381 (1975).

[6] D. Neuffer, IEEE Trans. Nucl. Sci. 26, No. 3, 3031 (1979).

[7] A. M. Al-khateeb et al., Phys. Rev. E 63, 026503 (2001). 
[8] T. J. P. Ellison et al., Phys. Rev. Lett. 70, 790 (1993).

[9] I. Hofmann, in Proceedings of EPAC94, London (World Scientific Publishing Co. Pte. Ltd., Singapore, 1994), p. 218.

[10] A. U. Luccio and W.T. Weng, in Workshop on SpaceCharge Physics in High Intensity Hadron Rings, AIP Conf. Proc. No. 448 (AIP, New York, 1998).

[11] I. N. Meshkov, Phys. Part. Nucl. 25, 6 (1994).
[12] A.W. Chao, Physics of Collective Beam Instabilities in High Energy Accelerator (Wiley, New York, 1993).

[13] B. Zotter, CERN Report No. CERN-85-19, 1985, p. 253.

[14] B. Zotter and F. Sacherer, CERN Report No. CERN-7713, 1977, p. 175.

[15] L. R. Evans, CERN Report No. CERN-84-15, 1984, p. 319. 